\title{
Manejo quirúrgico de las malformaciones arteriovenosas durales craneales. Serie de seis casos
}

M. Rivero-Garvía; G. Rodríguez-Boto; A. Pérez-Zamarrón; R. Gutiérrez-González; H. Zimman-Mansfeld; T. De Miguel y C. Saldaña-Galán

Hospital Clínico San Carlos. Madrid.

\section{Resumen}

Objetivos. En este artículo describimos la experiencia quirúrgica de nuestro centro en el manejo quirúrgico de las malformaciones arteriovenosas durales intracraneales (MAVd's).

Material y métodos. Presentamos una serie de seis casos, dos mujeres y cuatro hombres, con edades comprendidas entre 40 y 68 años, en el periodo que transcurre entre los años 2001 y 2006, en el Hospital Clínico San Carlos de Madrid.

Resultados. Cuatro de los seis casos fueron ingresados a través del servicio de Urgencias por clínica deficitaria (en dos casos) o disminución del nivel de conciencia (en dos pacientes); los dos restantes fueron remitidos desde consultas externas por cefalea de larga evolución y alteraciones en el estudio de neuroimagen sugerentes de FAVd o MAVd. En todos ellos el tratamiento quirúrgico fue definitivo.

Conclusiones. A pesar las múltiples opciones terapéuticas, la cirugía es de elección en MAVd que presenta especial tendencia a comportarse de forma agresiva; fundamentalmente con riesgo de hemorragia intracraneal.

PALABRAS CLAVE: Malformaciones arteriovenosas durales. Fístulas arteriovenosas durales. Hemorragia intracraneal. Tratamiento quirúrgico.

Surgical management of dural arteriovenous fistulae in six patients

Summary

Objetives. In this article, we describe our experience in surgical management of dural arteriovenous fistulae (dAVF).

Materials and methods. From Agust 2001 to Febrery 2006 a total of six patients, were admitted at our hospi-

Recibido: 15-12-09. Aceptado: 22-05-07 tal, with ages between 40 and 68 years.

Results. Four of the six cases were entered through the service of Emergency Service by neurological defecit (in two cases) or decrease in the level of consciousness (in two patients); the remaining two patients were refered by lengthy headache and alterations on neuroimagen studies suggestive of dAVF. All of them showed dAVF in different locations which were treated successfully with surgery of the unique mortality of treatment after angiographical studies.

Conclusion. Although multiple therapeutic options are avaible, surgery is in $\mathrm{dAVF}$ which shows aggressive clinical course, especially intracranial hemorrhage.

KEY WORDS: Dural arteriovenous malformation. Dural arteriovenous fistulae. Intracranial hemorrhage. Surgical treatment.

Introducción

Las fístulas arteriovenosas durales (FAVd) son conexiones directas entre arterias y venas (senos durales o venas corticales) sin red capilar intermedia localizadas en el interior de las capas de la duramadre, aunque ocasionalmente se observa un pequeño nidus intradural, denominándose entonces malformaciones arteriovenosas (MAVd). Constituyen entre el 10 al $15 \%$ de todas las conexiones arteriovenosas anómalas intracraneales ${ }^{1,10,11}$. A pesar de estar descritos, en la literatura, casos de cierre espontáneo en fístulas de bajo flujo ${ }^{2,4,5,8,10}$, su diagnóstico se basa en los síntomas neurológicos secundarios a: el efecto masa, el fenómeno de robo, la hipertensión venosa, su capacidad irritativa sobre la corteza cerebral o los efectos devastadores de su sangrado. Según su localización y su drenaje venoso se clasifican según orden decreciente de frecuencia en: del seno transverso-sigmoide, del seno cavernoso, del tentorio, del seno sagital-convexidad dural, de la fosa craneal anterior y de la región silviana. No obstante, las clasificaciones más importante se realizan por el reclutamiento de venas piales y sus anomalías morfológicas según Borden et al o Djindjian con la modificación de Cognard (tabla 1), pues 
Tabla 1

Resumen de los casos

\begin{tabular}{cccc}
\hline Casos & Sexo / Edad & Clínica inicial & Cirugía de urgencia \\
\hline 1 & Mujer / 40 & GCS 15 & No \\
\hline 2 & Varón / 55 & GCS 3S midriasis izquierda & Sí, evacuación de hematoma \\
\hline 3 & Varón / 65 & GCS 14 & No \\
\hline 4 & Varón / 50 & GCS 3 & Sí, evacuación de hematoma \\
\hline 5 & Mujer / 42 & GCS 14 & No \\
\hline 6 & Varón / 68 & GCS 14 & No \\
\hline
\end{tabular}

Tabla 2

Localización, descripción anatómica y clasificación de las MAVd de nuestra serie

\begin{tabular}{|c|c|c|c|c|c|c|}
\hline Caso & Sitio & Arterias aferentes & Drenaje venoso & $\begin{array}{c}\text { Trombosis } \\
\text { senos }\end{array}$ & Hemorragia & Tipo \\
\hline 1 & $\begin{array}{l}\text { Fosa craneal } \\
\text { anterior } \\
\text { derecha }\end{array}$ & $\begin{array}{l}\text { Etmoidales, } \\
\text { bilaterales oftálmicas } \\
\text { y esfenopalatinas }\end{array}$ & $\begin{array}{l}\text { Venas cerebrales } \\
\text { internas al seno recto y } \\
\text { transverso derecho }\end{array}$ & No & No & IV \\
\hline 2 & $\begin{array}{l}\text { Seno } \\
\text { longitudinal } \\
\text { superior } \\
\text { (tercio } \\
\text { posterior) }\end{array}$ & $\begin{array}{l}\text { Aporte bilateral de } \\
\text { ambas meníngeas } \\
\text { medias }\end{array}$ & $\begin{array}{l}\text { Venas corticales a los } \\
\text { senos: longitudinal } \\
\text { superior, recto y } \\
\text { transverso-sigmoideo } \\
\text { izquierdo }\end{array}$ & No & $\mathrm{Si}$ & IV \\
\hline 3 & $\begin{array}{l}\text { Convexidad } \\
\text { occipital } \\
\text { izquierda }\end{array}$ & $\begin{array}{l}\text { Occipital izquierda y } \\
\text { de ramas meníngeas } \\
\text { de ambas vertebrales }\end{array}$ & $\begin{array}{l}\text { Vena pial al seno sagital } \\
\text { superior }\end{array}$ & No & No & III \\
\hline 4 & $\begin{array}{l}\text { Convexidad } \\
\text { frontal } \\
\text { izquierda }\end{array}$ & $\begin{array}{l}\text { Temporal externa y } \\
\text { etmoidal izquierda }\end{array}$ & $\begin{array}{l}\text { Vena pial al seno } \\
\text { cavernoso }\end{array}$ & No & $\mathrm{Si}$ & IV \\
\hline 5 & $\begin{array}{l}\text { Seno } \\
\text { transverso- } \\
\text { sigmoide } \\
\text { derecho }\end{array}$ & $\begin{array}{l}\text { Arteria occipital derecha, } \\
\text { auricular posterior } \\
\text { derecha y arteria de } \\
\text { Bernasconi derecha }\end{array}$ & $\begin{array}{l}\text { Seno transverso } \\
\text { sigmoide derecho, seno } \\
\text { recto y venas corticales } \\
\text { al seno sagital superior }\end{array}$ & $\mathrm{Si}$ & $\mathrm{Si}$ & $\mathrm{IIa}+\mathrm{b}$ \\
\hline 6 & Tentorio & $\begin{array}{l}\text { Arterias occipitales } \\
\text { bilaterales, ramas } \\
\text { meníngeas de ambas } \\
\text { vertebrales y arteria de } \\
\text { Bernasconi derecha }\end{array}$ & $\begin{array}{l}\text { Vena única al plexo } \\
\text { venoso peritorcular }\end{array}$ & No & $\mathrm{Si}$ & III \\
\hline
\end{tabular}

nos informan de la agresividad de las mismas y de la necesidad de su tratamiento ${ }^{1,2,4}$.

Aunque las opciones terapéuticas son amplias (embolización arterial o venosa, radiocirugía o cirugía, solas o combinadas), el objetivo es único: efectuar un cierre completo de la lesión para evitar sangrados posteriores. El tratamiento de elección debe ser quirúrgico para las localizaciones accesibles, sobre todo en aquellas fístulas o malformaciones que ya hayan sangrado previamente ${ }^{2-5,8}$.

\section{Material y métodos}

Presentamos una serie consecutiva de seis pacientes, desde Agosto del año 2001 hasta Febrero del año 2006, 
Tabla 3

clasificación de Cognard y cols

\begin{tabular}{c|c}
\hline Tipo & Drenaje Venoso \\
\hline I & Flujo anterógrado hacia un seno dural \\
\hline II & $\begin{array}{c}\text { Drenaje hacia seno dural con reflujo a: } \\
\text { IIa: sólo al seno }\end{array}$ \\
& $\begin{array}{c}\text { IIb: sólo a venas corticales } \\
\text { IIa + b: tanto al seno como a las venas corticales }\end{array}$ \\
\hline III & Drenaje directo a venas corticales sin ectasia \\
\hline IV & Drenaje a venas corticales con ectasia venosa* \\
\hline V & Drenaje a venas leptomeníngeas perimedulares \\
\hline
\end{tabular}

$(*)>$ Calibre mayor de $5 \mathrm{~mm}$ o mayor de 3 veces su diámetro normal.

ingresados en nuestro hospital, bien a través del servicio de urgencias, bien a través de consultas externas. Todos los pacientes fueron sometidos a una angiografía cerebral selectiva de cuatro vasos diagnóstica previa a la oclusión quirúrgica de la MAVd.

\section{Resultados}

Nuestra serie está formada por seis pacientes, dos mujeres y cuatro hombres, con edades comprendidas entre 40 y 68 años. Cuatro de los seis casos fueron ingresados a través del servicio de Urgencias por clínica deficitaria (en dos casos) o disminución del nivel de conciencia (en dos pacientes); los dos restantes fueron remitidos desde consultas externas por cefalea de larga evolución y alteraciones en el estudio de neuroimagen sugerentes de FAVd o MAVd (tablas 2 y 3 ).

Caso 1: mujer de 40 años, con antecedentes de cefaleas de características no uniformes desde la juventud, remitida a consultas por un cuadro subagudo de alteración de la visión en ojo derecho (defecto visual inferior en campimetría de ojo derecho). En la TC craneal se observaba una lesión tubular frontobasal medial derecha (figura 1). La angioRM y la angiografía cerebral confirmaron la existencia de una MAVd de la fosa craneal anterior, dependiente de las arterias etmoidales procedentes de ambas arterias oftálmicas dilatadas; el drenaje venoso se realizaba a través de dos venas: una vena ectásica pial subfrontal que comunicaba con las venas cerebrales internas y el seno recto y otra, no dilatada, al seno transverso derecho (figura 2).

La paciente fue sometida a tratamiento electivo quirúrgico donde se realizó coagulación de vasos aferentes y venas de drenaje, con resolución completa de la MAVd. No se observó mejoría del defecto campimétrico tras la cirugía.

Caso 2: varón de 55 años que acude a Urgencias por pérdida brusca de conocimiento $(\mathrm{CGS}=3)$ y midriasis izquierda, precedida de cefalea, náuseas y vómitos. En la TC craneal se halló hemorragia subaracnoidea (HSA) en cisternas basales y extenso hematoma subdural agudo (HSDA) izquierdo, por lo que precisó evacuación urgente del hematoma (figuras 3 y 4). En la angiografía cerebral se detectó una MAVd del tercio posterior del seno longitudinal, con aferencias arteriales de ambas carótidas externas a través de las arterias meníngeas, y drenaje venoso a los senos longitudinal superior, recto y transversosigmoideo izquierdo a través de venas piales con aneurisma venoso asociado de $2 \mathrm{~cm}$ (figura 5).

Durante la cirugía programada para la resección de la MAVd, el paciente presentó una embolia pulmonar que obligó a suspender la cirugía, y a realizarla en un segundo tiempo, resecando completamente la lesión.

Caso 3: varón de 65 años, hipertenso, diabético no insulinodependiente, tratado con cirugía, radioterapia y quimioterapia por un tumor espermático. Debutó con un cuadro de amnesia global transitoria. En la TC craneal y RM

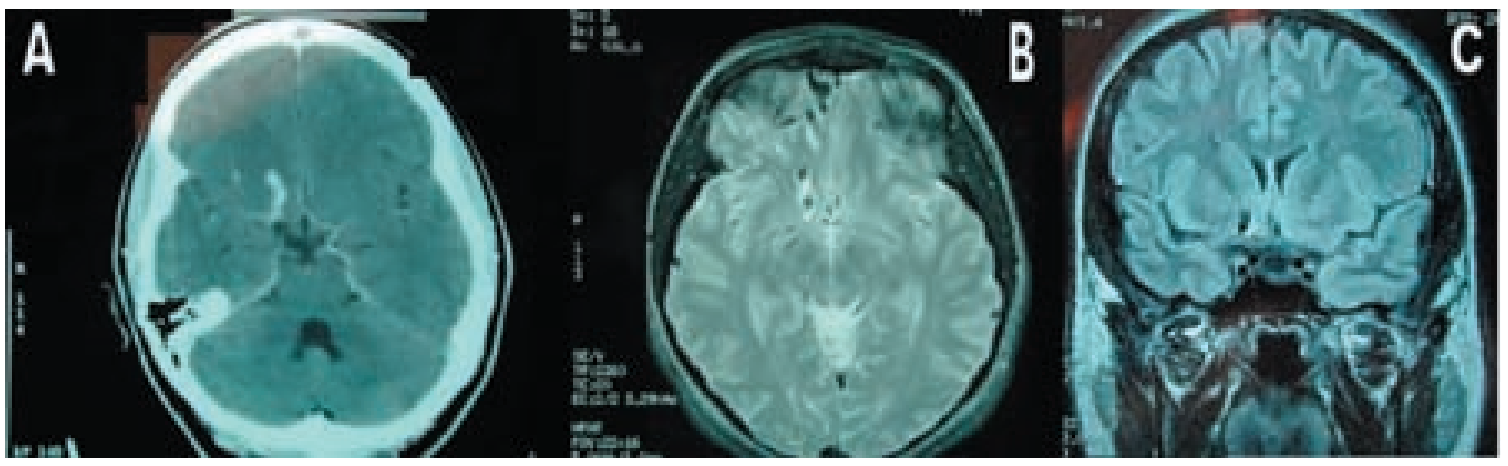

Figura 1. A: TC craneal con contraste en el que obserba una vena de drenaje anómala en la región medial y posterior en la fosa craneal anterior. B y C: secuencia T2 y FLAIR de la RM cerebral donde se observa la vena de drenaje y la ausencia de nidos de la FAVd. 


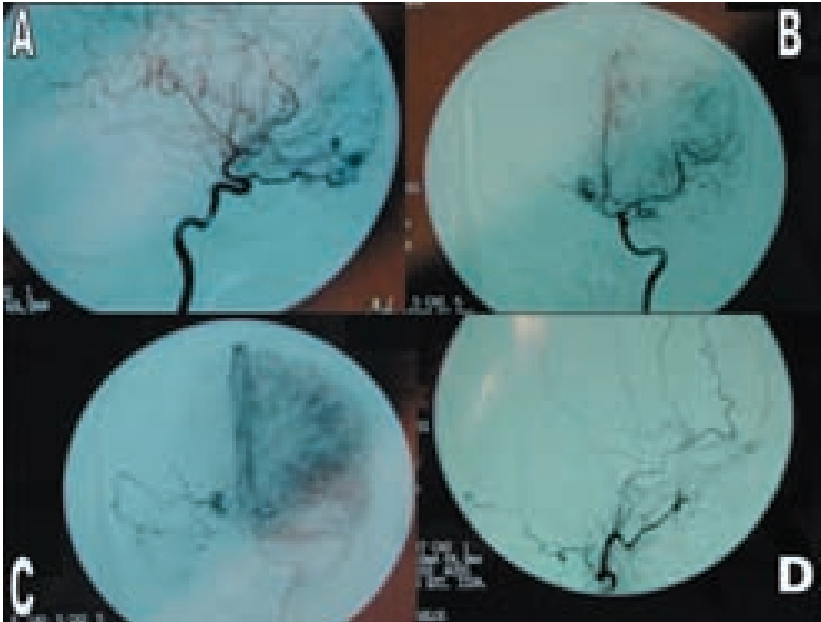

Figura 2. Aretiogragía prequirúrgica del caso 1, en la que se observa una FAVd con aportes arteriales de ambas oftálmicas, etmoidales y esfenopalatinas (A, B y D) y drenaje al seno transverso derecho y por las venas cerebrales internas al seno recto.

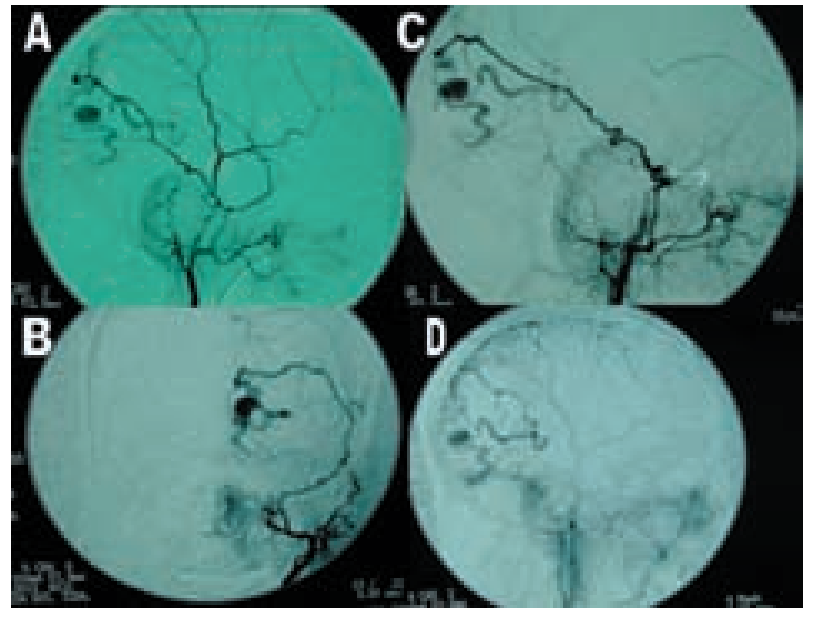

Figura 5. Aretriogragía diagnóstica del caso 2, donde se comprueba la existencia de una MAVd del seno longitudinal superior con aporte de ambas arterias meníngeas medias (fotos Ay B), y su drenaje a través de una vena única con una aneurisma venoso asociado a los senos.

Figura 3. Caso 2, en la TC craneal sin contraste (A) se observa una lesión hemorrágica occipital izquierda. Tras la utilización de contraste se observa en la imagen $B$ una vena de drenaje anómala.
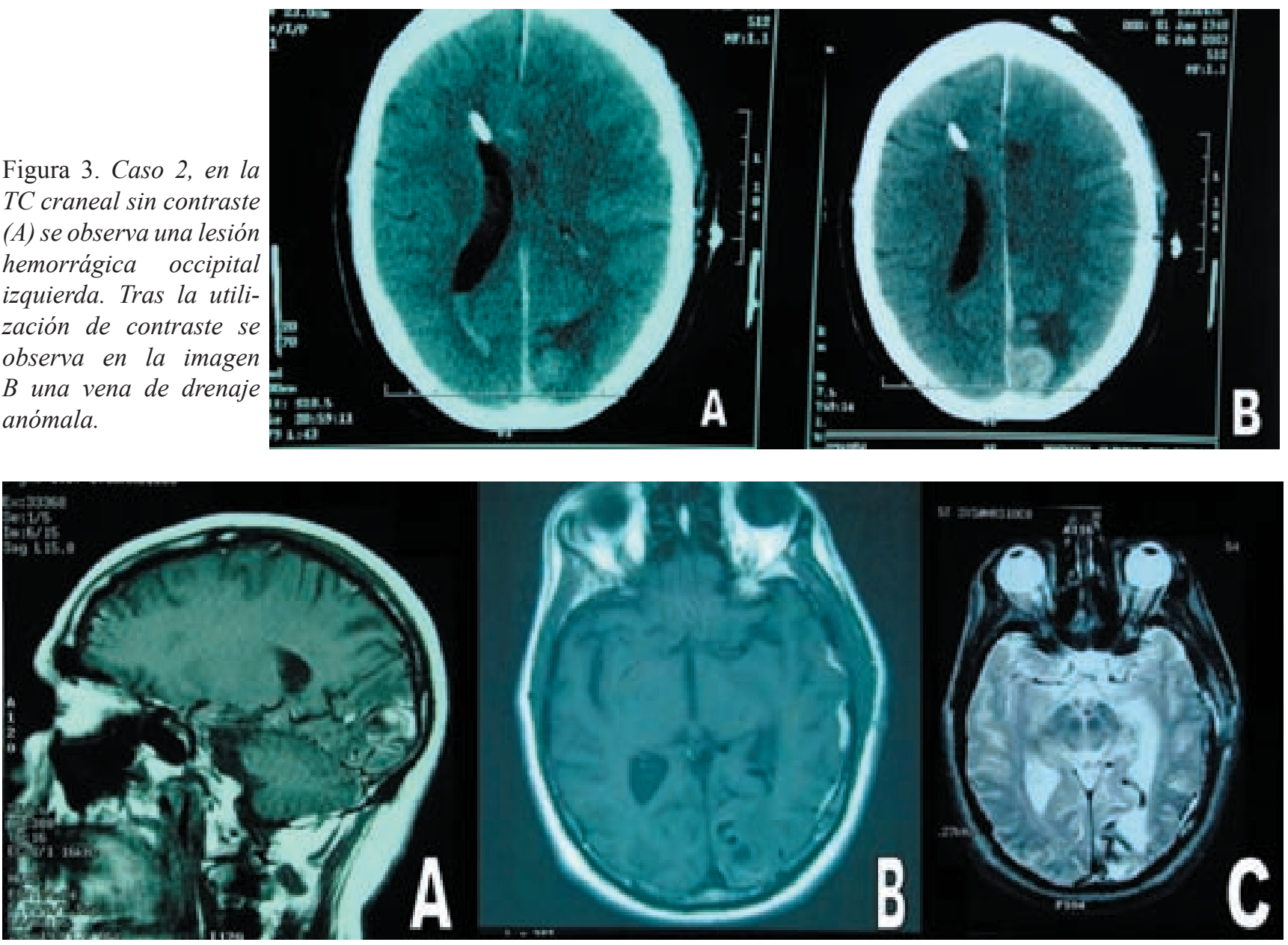

Figura 4. RM cerebral prequirúrgica del caso 2 donde se observa la vena anómala de drenaje occipital izquierda de la MAVd. 


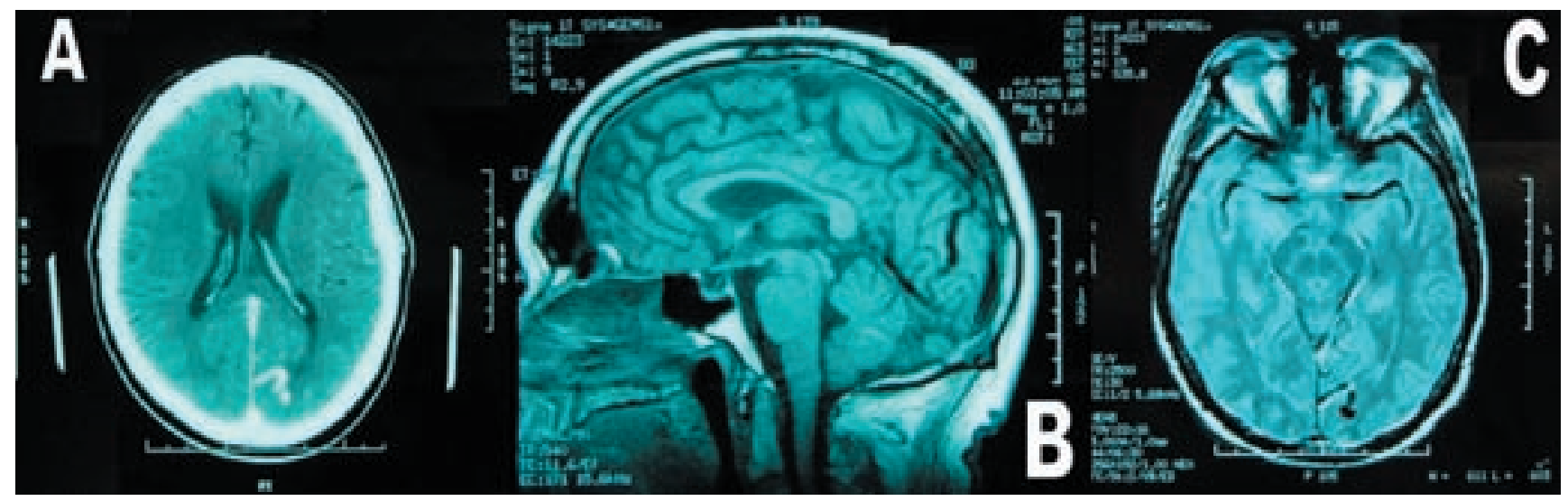

Figura 6. Detalle de la vena de drenaje del caso 3 en la TC con contraste (A), y el RM cerebral, sin que se observe nidos (B y C).

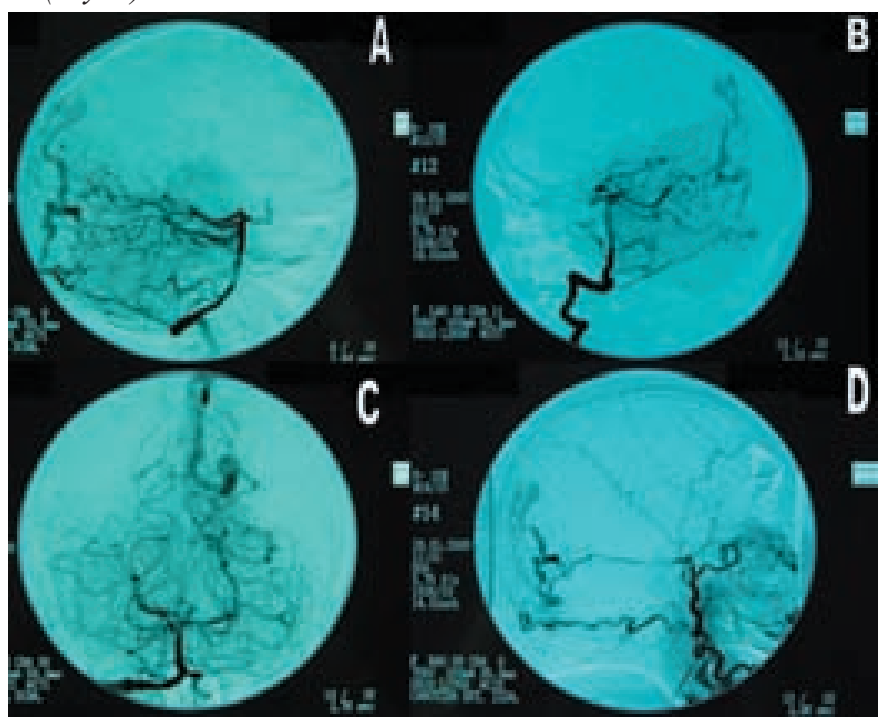

Figura 7. Angiografia prequirúrgica del caso 3 en el que se observa una FAVd con aporte arterial a través de ramas meningeas de ambas vertebrales $(A, B$ y $C$ ) y de la arteria occipital superficial (D). El drenaje se realiza a través de una vena tortuosa al se longitudinal posterior. cerebral se observó una vena de drenaje anómala occipital izquierda. La angiografía confirmó una MAVd con nidus adyacente a la tórcula, aferencias de ramas de la arteria occipital izquierda y de ambas meníngeas de ambas arterias vertebrales (figura 6). El drenaje venoso se realizaba a través de vena leptomeníngea única al tercio posterior del seno sagital superior (figura 7).

Tras la resección del nidus y la coagulación de las aferencias y la vena de drenaje, se observó la oclusión completa de la MAVd en la angiografía de control, sin secuelas clínicas para el paciente (figura 8).

Caso 4: varón de 50 años, remitido desde un hospital comarcal por cuadro de cefalea brusca con vómitos y disminución del nivel de conciencia hasta GCS de 3, secundario a HSDA hemisférico izquierdo con efecto masa que se evacuó con carácter de urgencia (figura 9). En los estudios de imagen posteriores (RM cerebral y angiografía) se detectó una FAVd de la convexidad frontal izquierda con aferencias de la arteria temporal superficial y rama etmoidal izquierda y drenaje venoso a través de una vena pial con dos aneurismas venosos asociados, que drenaba al

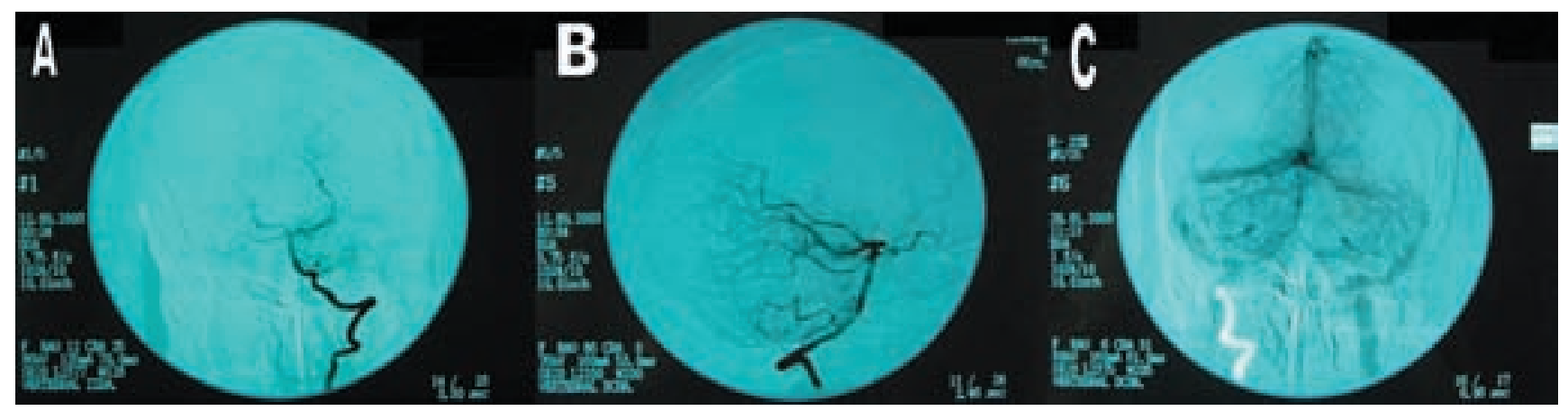

Figura 8. Angiografía posquirúrgica del caso 3, en la que se observa la correcta oclusión de la FAVd. 


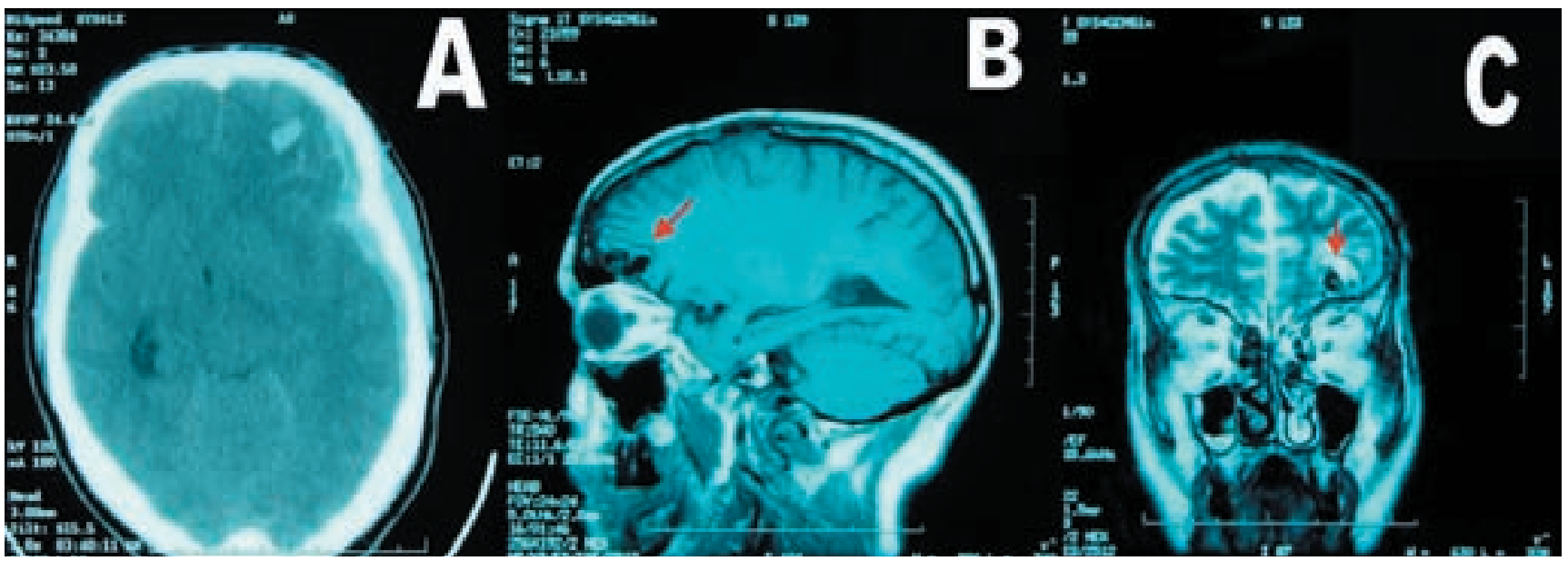

Figura 9. Caso 4, en la TC craneal (A) se observa un hematoma subdural agudo frontotemporal izquierdo con efecto masa. En la RM cerebral destacan dos aneurismas venosos asociados al avena de drenaje (flecha en $B$ y C).

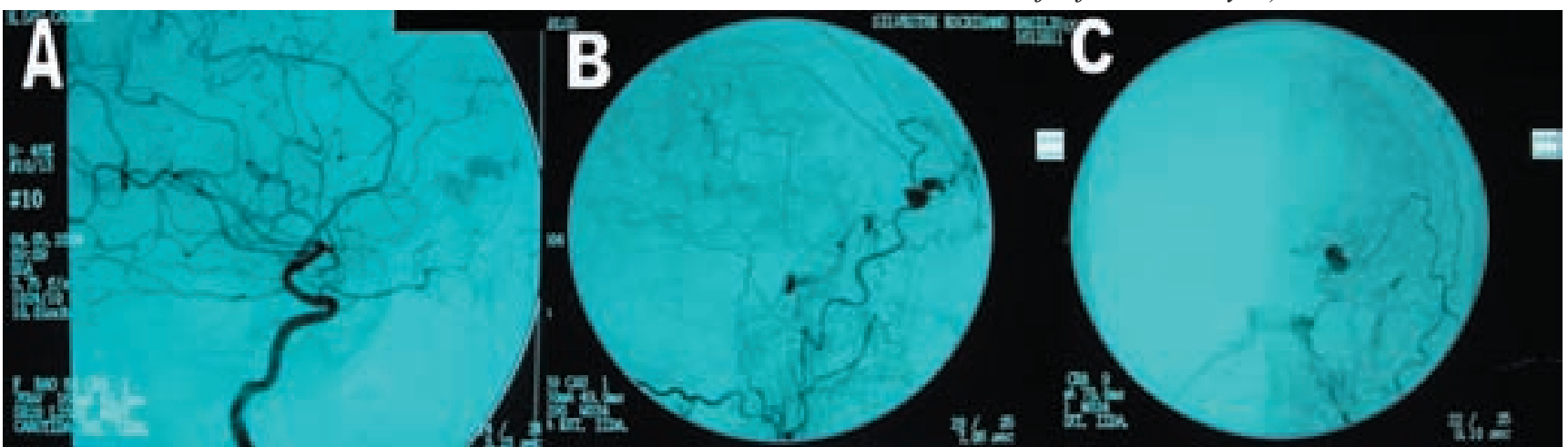

Figura 10. Angiografía diagnóstica del caso 4 donde se observa MAVd con aporte de la arteria oftálmica (A), de la arteria temporal superficial (B) y su drenaje a través de de una vena única con dos aneurismas asociados al seno cavernoso (B y C).

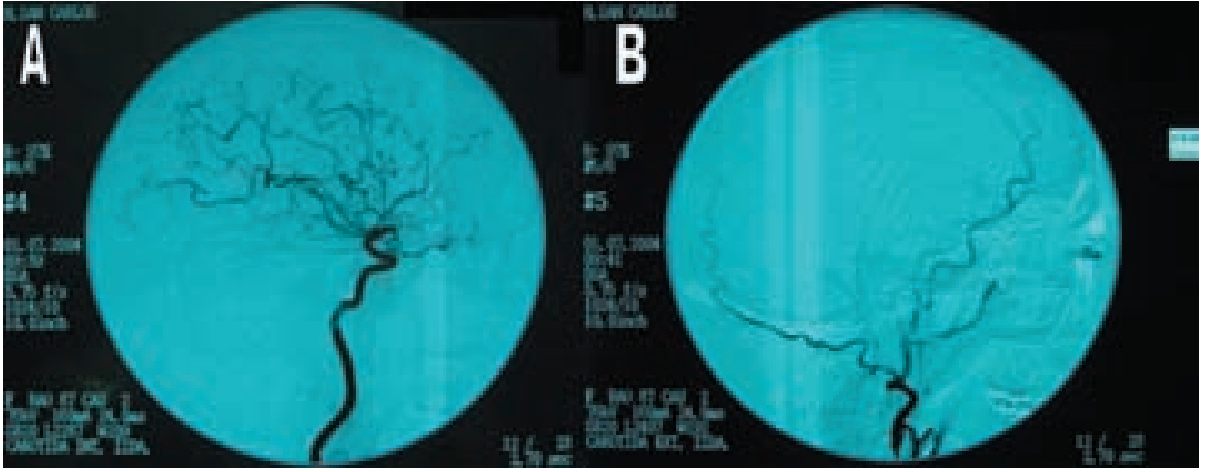

Figura 11. Angiografía de control del caso 4 en la que no se observa resto de la MAVd

seno cavernoso (figuras 9 y 10).

Tras el clipaje transitorio de la vena de drenaje preaneurismática a su salida de la MAVd, se realiza resección de ambos aneurismas y coagulación de aquella, retirando el clip vascular sin incidencias con cierre completo de FAVd en la angiografía de control (figura 11).

Caso 5: mujer de 42 años, que ingresó en el Servicio de urgencias por cefalea resistente a tratamiento analgésico habitual, acompañada de afasia mixta, alexia, náuseas y vómitos. La paciente estaba diagnostica previamente de migrañas típicas y tinnitus sincrónico con el pulso desde hacía un año, que podía oírse sin necesidad de fonendoscopio en región retromastoidea derecha. En la TC craneal se observó un hematoma intraparenquimatoso en región temporal derecha (figura 12). En la angioRM cerebral se evidenció una FAVd en región temporal derecha, que se confirmó en la angiografía cerebral (figuras 13 y 14). La MAVd asentaba en la pared del seno transverso-sigmoideo derecho, sus aferencias procedían de la arteria occipital 


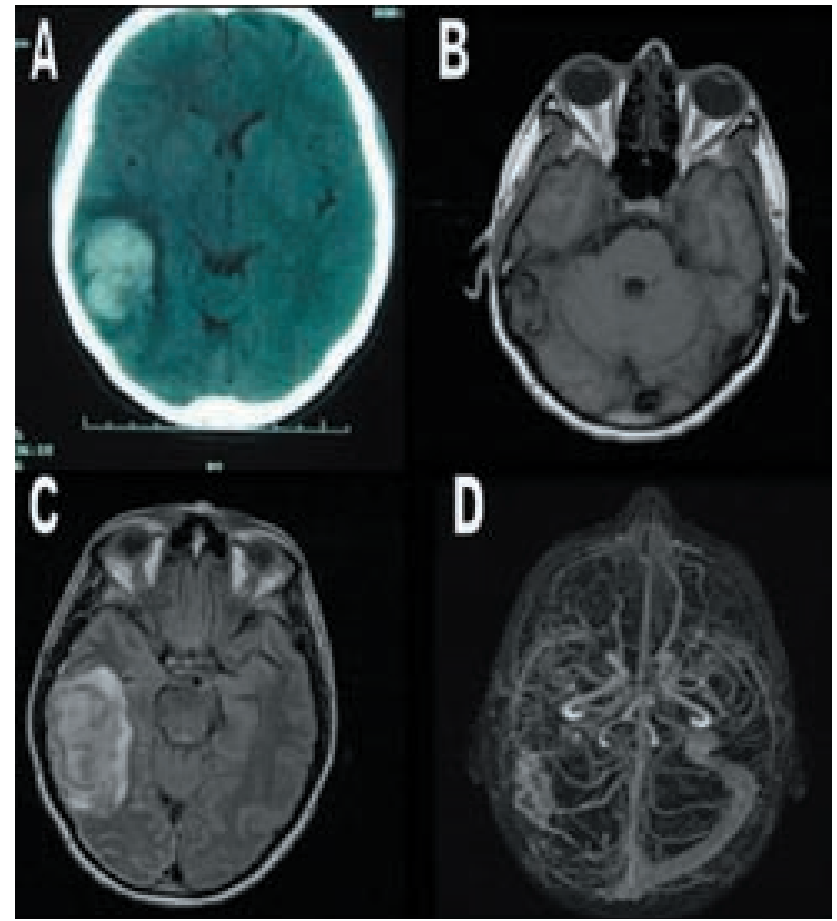

Figura 12. En la foto $A$ y $C$ se observa el hematoma parietal derecho del caso 5. En la imagen $B$ se observa el lecho de la MAVd. La foto D corresponde a la angioRM en el que se obserba el nidos de la MAVd y la trombosis del seno transverso-sigmoideo derecho.

derecha, auricular posterior derecha y rama meníngea tentorial de la carótida derecha (arteria de Bernasconi). El drenaje se realizaba al seno transverso-sigmoideo derecho y seno recto, así como al seno longitudinal superior a través de venas corticales. Tanto el seno transverso derecho como la parte superior de la vena yugular derecha no eran permeables en la fase venosa de la angiografía (figura 14).

Tras la resección en bloque de FAVd y ligadura del seno transverso proximal y distal a la misma se observó, en la angiografía de control, desaparición completa de la misma (figura 15).

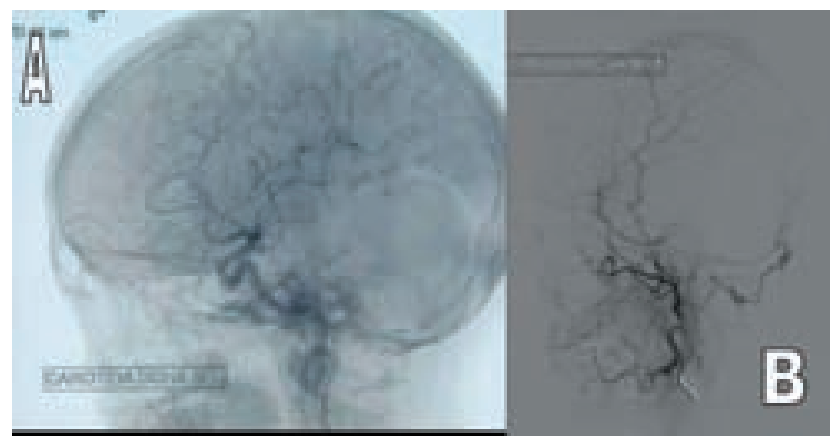

Figura 14. Angiografía de control del caso 5 en la que se observa la correcta resolución de la MAVd del seno transverso-sigmoide derecho.

Caso 6: varón de 68 años, con clínica inicial súbita de nucalgia, rigidez de nuca e inestabilidad de la marcha. En la TC se observó una HSA en cisternas basales y hematoma cerebeloso derecho (figura 16). La angioRM y angiografía cerebral descubrieron una FAVd en fosa posterior, con aferencias de ambas arterias occipitales, ramas meníngeas de sendas vertebrales y arteria tentorial de Bernasconi derecha y drenaje a través de una única vena tortuosa que salía del tentorio y llegaba al plexo venoso peritorcular (figuras 17 y 18).

Se colocó un clip permanente en la vena de drenaje con oclusión completa de FAVd en la angiografía de control (figura 19). El paciente presentó un infarto isquémico parietal derecho en el postoperatorio inmediato que le causó una hemiplejía izquierda residual.

\section{Discusión}

Las MAVd's representan epidemiológicamente del 10\% al $15 \%$ de todas las MAVs intracraneales. Su localización más frecuente es el seno transverso sigmoide, seguidas de las del seno cavernoso. Su distribución por sexos depende de su localización, las del seno transverso-sigmoide y las del seno cavernoso son más frecuentes en mujeres, mien-

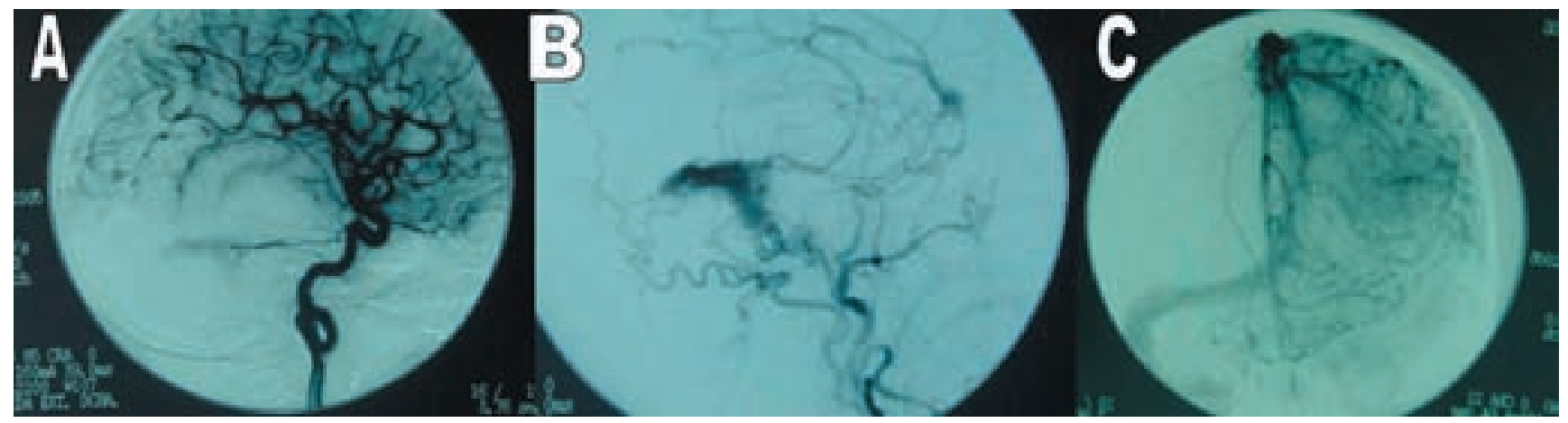

Figura 13. En la angiografía del caso 5 se observa como la MAVd sufre aporte arterial dela arteria de Bernasconi (A) y de la carótida externa derecha (B). El seno trn;ansverso-sigmoidey la yugular derecha se encontraban trombosadas (C). 

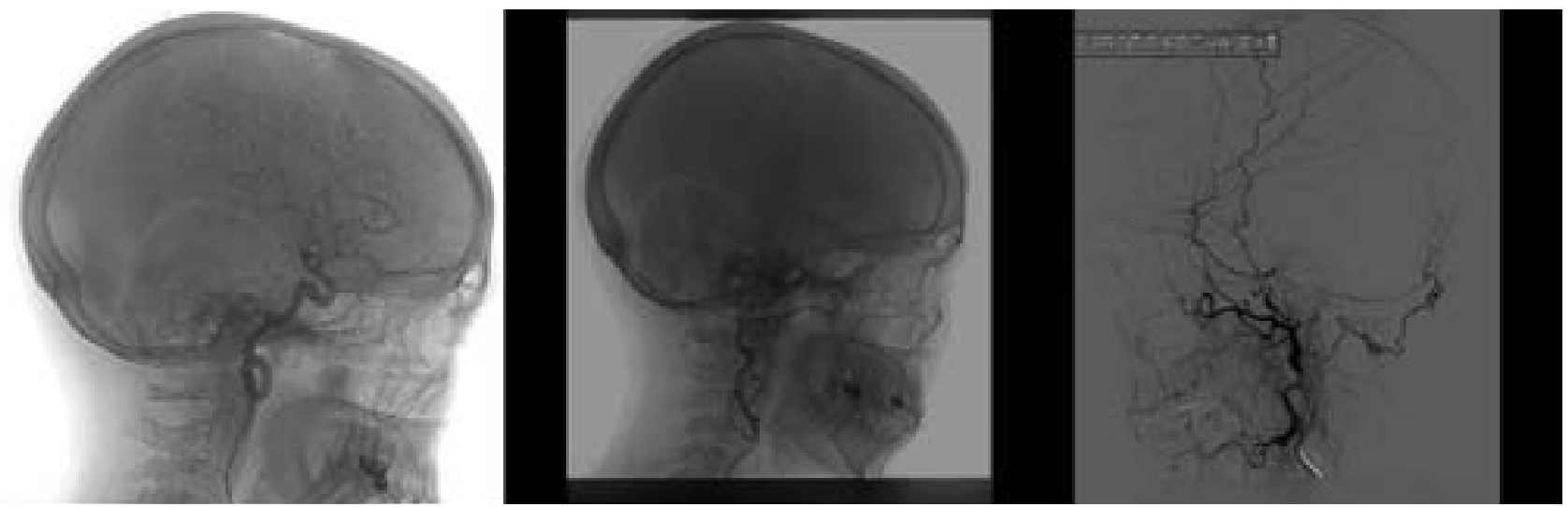

Figura 15. Angiografía de control del caso 5, arteria carótida izquierda (imagénes izquiera y central) y carótida externa derecha (imagen derecha) donde no se observa resto de la MAVd.

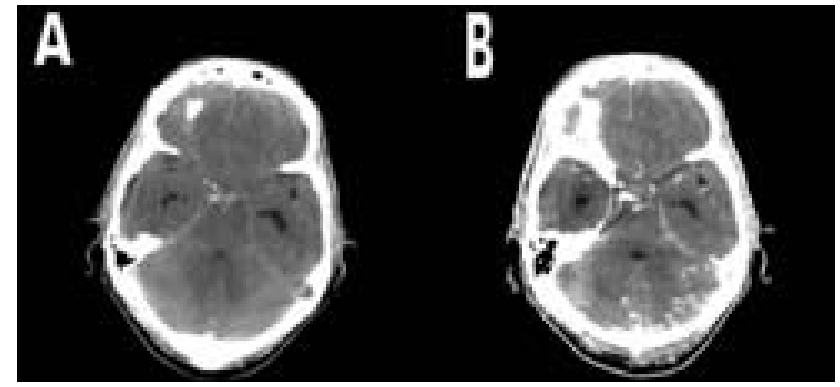

Figura 16. Caso 6: en la TC de urgencias se observa un aumento de la densidad de fosa posterior por HSA (A), la administración de contraste pone de manifiesto la existencia de vasos anormales (B).

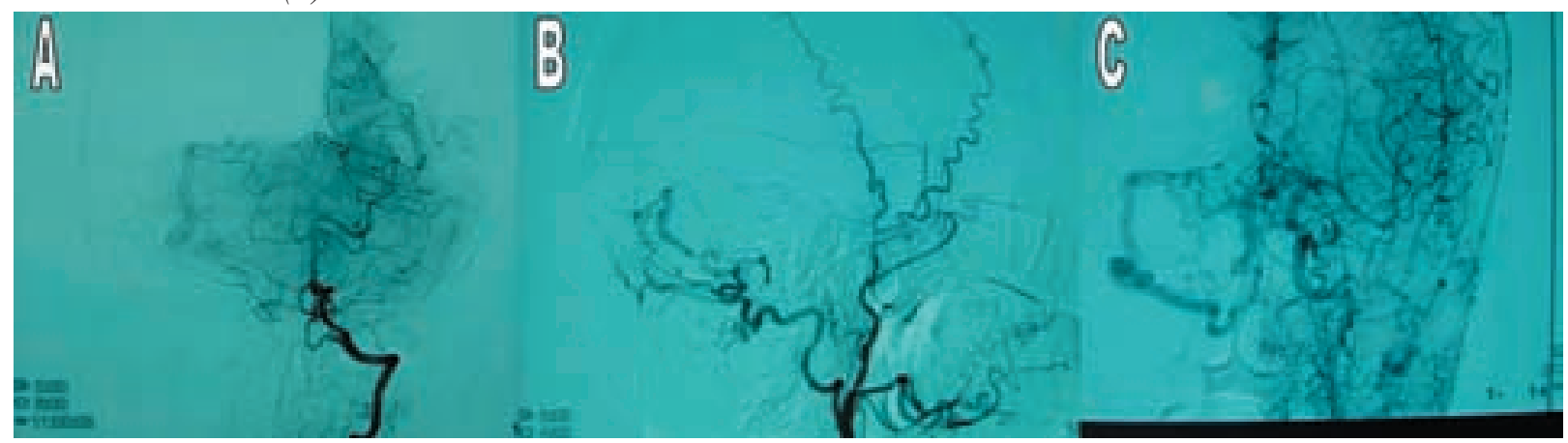

Figura 18. Angiografia cerebral del caso 6 prequirúrgica, donde se observa FAVd de la fosa posterior con una vena de drenaje única al plexo peritorcular.

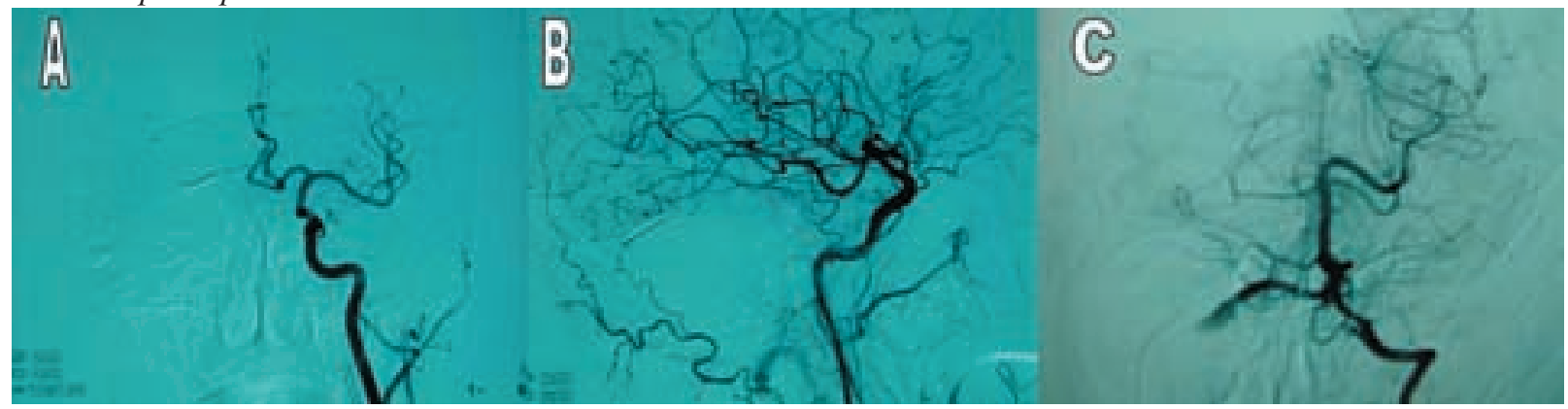

Figura 19. Angiografía de control en la que no se observa resto de la FAVd. Se puede ver el clip sobre la vena de drenaje (flecha negra). 
tras que las del seno longitudinal superior y las de la hoz son más frecuentes en hombres ${ }^{3,9}$.

La etiopatogenia de las MAVd es, actualmente, desconocida. Entre las distintas causas se mencionan en la literatura: su carácter congénito, traumatismos previos, trombosis de senos durales con reclutamiento de microfístulas arteriovenosas o agresiones quirúrgicas ${ }^{3}$.

La historia natural de las FAVd presenta un amplio abanico de posibilidades, desde el cierre espontáneo hasta un sangrado devastador ${ }^{1,4,5}$. La agresividad de las mismas depende de su drenaje, es decir, si el vaso eferente es capaz de asumir el torrente circulatorio sin que suponga un "stress" excesivo sobre su pared y conlleve la rotura del mismo. Por eso las fístulas que drenan directamente en los senos durales presentan un curso más indolente, que aquéllas que lo hacen a través de venas piales ${ }^{2-5}$. Este hecho repercute tanto en su clasificación como en la elección del tratamiento pues tratamientos parciales aumentan el riesgo de resangrado al reclutar vasos de calibre menor.

La clasificación propuesta por Djindjian y cols. en 1977 y posteriormente modificada por Cognard y cols. ${ }^{4,6}$ (tabla 1) dividen las MAVd según su drenaje venoso en anterógrado normal (grado I) y aquéllas en las que su drenaje venoso sufre modificaciones que bien presentan reflujo (grado II), o reclutan venas corticales con pared más débil que la propia de los senos venosos (grados III y IV), o drenan a venas leptomeníngeas perimedulares (grado V). El riesgo de rotura es mayor cuanto menor es el calibre de la vena y mayor deformidad anatómica presenta (ectasia y aneurisma venoso). En nuestra serie, los casos 1, 2 y 4 presentaron una MAVd tipo IV, los casos 3 y 6 un tipo III y el caso 5 un tipo IIa+b. En los casos 2 y 4 además existían aneurismas venosos asociados.

Borden y cols. ${ }^{2}$, simplificaron la clasificación de Cognard y cols., en tres tipos de MAVd: el tipo I, que incluyen los tipos I y IIa de Cognard, en el que existe drenaje únicamente a un seno o una vena meníngea, ya sea anterógrado o retrógrado. El tipo II, que une a los tipos IIb y IIa+b, en el que el drenaje se realiza a un seno dural a la vez que retrógradamente a venas corticales o leptomeníngeas, y el tipo III, que aúna los tipos III, IV y V de Cognard, en el que el drenaje retrógrado es exclusivo a venas corticales o leptomeníngeas.

En ambas clasificaciones, el riesgo de un debut agresivo, es decir, de rotura o defecto neurológico, se produce en el $2 \%$ de los pacientes con MAVd tipo I, aumentando progresivamente al $39 \%$ en el Tipo II y al $79 \%$ en el Tipo III $^{2-6}$. En nuestra serie, los casos 2, 4, 5 y 6 presentaron un episodio hemorrágico como clínica inicial, con disminución del nivel de conciencia importante en los casos 2 y 4 , que precisaron con carácter de urgencia evacuación del hematoma ${ }^{2}$.

Un claro ejemplo de la importancia de la hemodinámica y el reclutamiento de venas piales normales es la paciente del caso 5. Dicha paciente refería acúfenos en el oído derecho de años de evolución (por las turbulencias del flujo que eran transmitidas por la vía ósea hasta la clócea). Sin embargo, en los días previos el tinnitus desapareció, hecho que podría haberse debido a la trombosis definitiva de la vena yugular y el seno transverso-sigmoideo ipsilateral, lo cual habría provocado que el flujo se desviara a venas piales de pared más débil, que acabaron rompiéndose a distancia de la FAVd.

Entre las múltiples opciones terapéuticas se encuentra la embolización transarterial que se reserva para casos con aferencias escasas y accesibles o en aquellos casos en los cuales la oclusión completa se puede obtener en pocas sesiones. En líneas generales su tasa de éxito no alcanza el $50 \%{ }^{3}$. En aquellas MAVd donde la dilatación varicosa es llamativa, como en le caso de las fístulas que afectan al seno cavernoso, la embolización transvenosa es otra opción a tener en cuenta, de hecho, esta técnica puede tener mayor rentabilidad terapéutica que la embolización arterial. La radiocirugía presenta el problema del cierre diferido en aquellas situaciones de sangrado agudo, y el aumento, por tanto, del riesgo de resangrado hasta el cierre definitivo de la MAVd si este llega a producirse. A pesar de ello, la utilización por separado o en combinación de estos tratamientos debería considerarse en aquellos casos en los que aún siendo sintomáticas las MAVd, sean inoperables tanto por el alto riesgo quirúrgico como por presentarse en enfermos de edad avanzada ${ }^{1,3,8,10,12}$.

La cirugía se puede realizar desde dos puntos de ataque: el sector arterial, mucho más farragoso y a veces ineficaz cuando las MAVd's presentan múltiples aferencias, y desde el sector venoso, en aquellas situaciones en las que no se identifica nidus y el drenaje es único ${ }^{5,7,8,12}$, de manejo mucho más sencillo y muy efectivo, como lo demuestran los casos 4 y 6 . La resección en bloque se debe realizar siempre que se identifique un nidus, como en los casos 1, 3 y 5.

Como conclusión, podemos decir que el tratamiento de las MAVd tipos I de Cognard, debe realizarse sólo en aquellos pacientes con síntomas atribuibles a la misma. En los tipos II, la embolización ha de ser considerada como una posibilidad eficaz ${ }^{1,3}$. Nuestra recomendación final es la de realizar un tratamiento quirúrgico de las MAVd con presentación agresiva (tipos III y IV), por la alta tasa de éxito que proporciona dicha técnica.

\section{Bibliografía}

1. Álvarez, R.F., Frutos, R., García, R.P., Isla, A., Pascual, J.M.: Malformaciones arteriovenosas durales intracraneales. Revisión de una serie de 38 casos. Actualización del diagnóstico y tratamiento. Neurocirugía 2000;11: 7-22. 
2. Borden, J.A., Wu, J.K., Shucart, W.A.: A proposed classification for spinal and cranial dural arteriovenous fistulous malformations and implications for treatment. J Neurosurg 1995; 82: 166-179.

3. Boto, G.R., Lobato, R.D., Campollo, J., Rivas, J.J.: Malformaciones arteriovenosas durales del seno longitudinal superior y la hoz cerebral. Presentación de cinco casos. Neurocirugía 1998; 9: 295-306.

4. Cognard, C., Gobin, Y.P., Pierot, L., et al.: Cerebral dural arteriovenous fistulas: clinical and angiographic correlation with a revised classification of venous drainage. Radiology 1995; 194: 671-680.

5. Collice, M., D'Aliberti, G., Arena, O., Solaini, C., Fontana, R.A., Talamonti G.: Surgical treatment of intracranial dural arteriovenous fistulae: role of venous drainage. Neurosurgery 2000 ; 47: 56-67.

6. Djiandjian, R., Merland, J.J., Theron, J.: Super-selective arteriography of the external carotid artery. New York; Springer-Verlag, 1977; pp. 606-628.

7. Kattner, K.A., Roth, T.C., Nardone, E.M., Giannotta, S.L.: The treatment of complex dural arteriovenous fistulae through cranial base techniques. Neurol India 2004; 52: 325-331.

8. Kiyosue, H., Hori, Y., Okahara, M., et al.: Treatment of intracranial dural arteriovenous fistulas: current strategies based on location and hemodynamics, and alternative techniques of transcatheter embolization. Radiographics 2004; 24:

\section{Comentario al trabajo: Manejo quirúrgico de las mal- formaciones arteriovenosas durales craneales. Serie de seis casos de M. Rivero Garvía y cols}

El Trabajo de Mónica Rivero y cols es una muestra de que la cirugía sigue siendo el tratamiento más efectivo para muchos paciente con patología neurovascular.

Las malformaciones o fístulas arteriovenosas durales (MAVD) tienen una presentación clínica muy variable, desde las que se curan espontáneamente hasta las que se
1637-1653.

9. Kubo, M., Kuwayama, N., Hirashima, Y., Kurimoto, M., Takaku, A., Endo, S.: Dural arteriovenous fistulae developing at different locations after resolution of previous fistulae: report of three cases and review of the literature. AJNR Am J Neuroradiol 2002; 23: 787-789.

10. Ng, P.P., Halbach, V.V., Quinn, R., et al.: Endovascular treatment for dural arteriovenous fistulae of the superior petrosal sinus. Neurosurgery 2003; 53: 25-33.

11. Raupp, S., van Rooij, W.J., Sluzewski, M., Tijssen, C.C.: Type I cerebral dural arteriovenous fistulas of the lateral sinus: clinical features in 24 patients. Eur J Neurol 2004 ; 11: 489-491.

12. Vougioukas, V.I., Coulin, C.J., Shah, M., Berlis, A., Hubbe, U., Van Velthoven, V.: Benefits and limitations of image guidance in the surgical treatment of intracranial dural arteriovenous fistulas. Acta Neurochir (Wien) 2006; 148: 145-153.

Rivero-Garvía, M.; Rodríguez-Boto, G.; Pérez-Zamarrón, A.; Gutiérrez-González, R.; Zimman-Mansfeld, H.; De Miguel, T.; Saldaña-Galán, C.: Manejo quirúrgico de las malformaciones arteriovenosas durales craneales. Serie de seis casos. Neurocirugía 2007; 18: 383-393.

Correspondencia postal: Mónica Rivero Garvía. Hospital Clínico San Carlos. C/ Profesor Martín Lagos s/n. 28040 Madrid

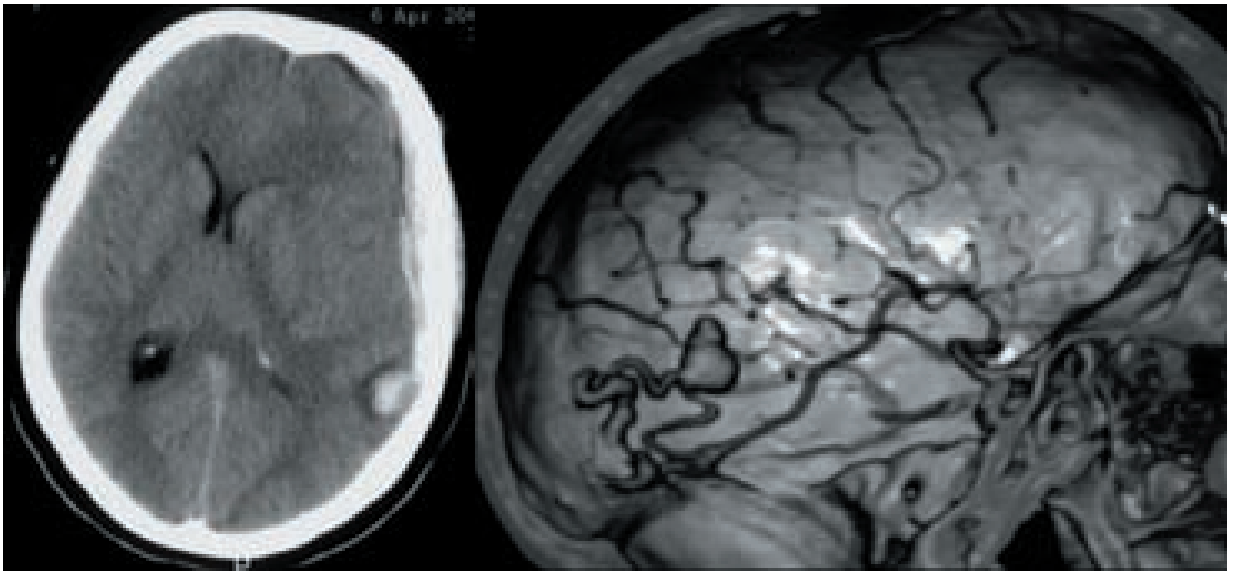

Figura 1. Paciente de 59 años que sus familiares la encontraron en coma $(G C S=6)$. El residente de guardia de neurocirugía pensó que el hematoma subdural agudo podía no ser traumático y el angio-TC demostró una FAVD. El tratamiento quirúrgico urgente permitió evacuar el hematoma y tratar la FAVD con buen resultado clínico y angiográfico 
cerebral y por el contrario el $40 \%$ y el $80 \%$ de las de tipo II y III respectivamente.

Las técnicas endovasculares evolucionan rápidamente pero los resultados son inferiores a los de la cirugía y no es excepcional que algunas MAVD se repermeabilicen y es extremadamente peligroso el tratamiento parcial de las MAVD que muestren un flujo retrógrado leptomeníngeo.

Nuestro conocimiento sobre esta patología ha aumentado mucho en los últimos años y sin duda con un abordaje multidisciplinario se consigue el mejor resultado terapéutico. Así pues, sería deseable que los especialistas no acostumbrados a esta patología refieran a estos pacientes preferentemente a neurocirujanos integrados en equipos multidisciplinarios, con interés por estas enfermedades antes que a otros especialistas que ofrecen tratamientos, teóricamente, menos agresivos pero menos eficaces.

Por último, no sabemos cuantas hemorragias intracraneales que operamos de urgencia pueden ser debidas a una MAVD, la angioTC puede ayudarnos en muchas ocasiones (Fig. 1), siempre que lo sospechemos, a tratar eficazmente, en un solo tiempo, a estos pacientes. 